\title{
EFFECT OF ASYMMETRIC ROLLING ON FORMABILITY OF PURE ALUMINIUM
}

\author{
Swagata Dutta ${ }^{1}$ and M. S. Kaiser ${ }^{2 *}$ \\ ${ }^{1}$ Institue of Appropriate Technology, ${ }^{2}$ Directorate of Advisory, Extension and Research Services \\ Bangladesh University of Engineering and Technology, Dhaka- 1000, Bangladesh \\ *Corresponding e-mail: mskaiser@iat.buet.ac.bd
}

\begin{abstract}
Uniaxial tensile test was carried out at different loading direction $\left(0^{\circ}, 90^{\circ}\right.$ and $\left.45^{\circ}\right)$ on rolled aluminium sheets. The anisotropic coefficient was analyzed to reveal formability characteristics. The shear strain that was imposed during the ASR was extensive and the shear marks was inclined more or less linearly without being zigzag or spiral lines. The ASRC improved the normal anisotropy coefficient compared to CR specimens. The highest normal anisotropy and the lowest planar anisotropy were observed by ASRC samples. Therefore, it was found that, asymmetric rolling especially ASRC can be used to improve the formability of aluminium thick sheets.
\end{abstract}

Keywords: Rolling, sheet, formability, texture, anisotropy.

\section{INTRODUCTION}

Forming industries have gained a number of ramifications over the years. There are different variants regarding forming of metals. Of them, rolling is one of the top used methods. As being successfully exploited in automotive industries as well as food packaging, forming of metals has become a common industrial practice over the years ${ }^{1-4}$. Aluminium alloys have gained interest in forming industries especially in the case of rolling due to its low density and high corrosion resistance. However, high production cost, low formability and low strength compared with other structural metallic material imposes certain constraints. This study was deliberately confined to asymmetric rolling of pure aluminium in order to investigate its effect on the mechanical properties. As aluminium and its alloys always suffer from limited formability issues, any improvement would be impulsive ${ }^{2-7}$. Asymmetric rolling is a special kind of sheet metal rolling process. Aluminium formability is dependent on the anisotropic coefficient as well as changes in the strain hardening that are imposed during plastic deformation. Anisotropic coefficient (R) is actually the width to thickness reduction ratio and can be represented by the following expression ${ }^{1-5}$.

$\mathrm{R}=\varepsilon_{22} / \varepsilon_{33}$

Here $\varepsilon_{22}$ is the true strain of the width and $\varepsilon_{33}$ is the true strain of the thickness which is given by the following expression [5]. If the coefficient ( $\mathrm{R})$ is high the formability of the sheet is high. This means the sheet is elongated more in the width than thickness direction before failure.

Where,

$\varepsilon_{22}=\ln \left(\mathrm{w} / \mathrm{w}_{0}\right) \& \varepsilon_{33}=\ln \left(\mathrm{t} / \mathrm{t}_{0}\right)$

Assuming the volume conservation the thickness change of the sheet can be estimated from the other two ${ }^{5}$.

$\varepsilon_{33}=-\left(\varepsilon_{11}+\varepsilon_{22}\right)$

Here $\varepsilon_{11}=\ln \left(1 / 1_{0}\right) ; 1=$ Final length; $1_{0}=$ Initial length In order to determine the mean anisotropy coefficient the R-values measured from different directions $(0,45$ and 90) are expressed by the following expression (eqn. 5) ${ }^{5}$. This coefficient is termed as normal anisotropy coefficient.

Normal anisotropy $(/ \mathrm{R})=\left(\mathrm{R}_{0}+\mathrm{R}_{90}+2 \mathrm{R}_{45}\right) / 4$

Planar anisotropy $(\Delta \mathrm{R})=\left(\mathrm{R}_{0}+\mathrm{R}_{90^{-}}-2 \mathrm{R}_{45}\right) / 2$

Increase in the normal anisotropy is related to the increase in the sheet formability, whereas, planar anisotropy is related to development of earring. For cases where $\Delta \mathrm{R} \neq 0$, chances are to form earrings. It was seen that developing shear texture component throughout the thickness of the sheet can induce the sheet to be more formable. Asymmetric rolling is one of the ways to obtain such properties. The formability depends not only on intrinsic factors but also on extrinsic factors ${ }^{1,2}$. Materials parameters as well as extrinsic parameters are the reason for different formality characteristics. Of the materials parameter crystallographic texture and grain size is of vital importance. On the other hand, in the case of extrinsic parameter change of strain path, strain rate change and forming temperature is of vital importance ${ }^{3}$. Crystallographic texture is an intrinsic feature. It resembles the preferential orientation of the grains in a polycrystalline. It is one of the major reason for which the material shows anisotropic behavior. There are various ways to represent crystallographic texture in materials systems and of them the representation by pole figure has been most regular practice over the years. Previous studies on this area were conducted on thin sheets. Investigation on the effect of asymmetric rolling on thick sheets was not studied compre- 
hensively. Hence, the present study aims at to study whether asymmetric rolling can be used to improve the formability pure aluminium thick sheets. Modeling the mechanical behavior of polycrystalline materials from the responses of their single crystals has been a challenging task. Polycrystals consist of grains which, due to mutual interaction, cannot deform freely as single crystals. The compatibility process of deformation between neighbor grains leads to the development of local accommodation stresses and to the differences between macroscopic and microscopic imposed strain/stress states. Of them, The Taylor model assumes that all the grains of the polycrystal undergo the same homogeneous strain as the polycrystal, keeping the continuity of the grain boundaries. Taking into account the crystallographic orientation of all grains of the polycrystal:

$\sigma=\langle M>\tilde{\tau}$

Where $<\mathrm{M}>$ is the average Taylor factor and $\tilde{\tau}$ is the average shear stress. The application of this model to simulate the polycrystal mechanical behavior is limited by the ambiguity in the selection of the activated slip systems, when more than one combination of five independent slip systems are solution.

\section{EXPERIMENTAL WORK}

In this study, commercially pure aluminium sheets of $8 \mathrm{~mm}$ thickness were used. The sheets were heated where the temperature rate was set to $10^{\circ} \mathrm{C} / \mathrm{min}$ and then the holding time was 1 hour at $400^{\circ} \mathrm{C}$. These sheets were allowed to cool in the furnace for about 1 hour and then were removed from the furnace and cooled naturally in air. This was done to develop a recrystallized state. All the sheets were heat treated in the same manner. After recrystallization, the sheets were rolled both conventionally and asymmetrically. The rolls have diameters of about $180 \mathrm{~mm}$ ensuring similar upper and lower cylinders. The speed of the rolls was controlled by dedicated software. Asymmetric rolling was done where the lower rolls had $5 \mathrm{rpm}$ and the upper rolls had $15 \mathrm{rpm}$. For conventional rolling the speed of the both rolls were kept constant at $15 \mathrm{rpm}$. The thickness reduction per pass was kept constant at $15 \%$. Conventional rolling (CR) was always conducted in the same direction whereas the asymmetric rolling (ASR) were conducted in two ways. In the asymmetric rolling continuous (ASRC) the rolling direction was not altered. For the asymmetric rolling reverse (ASRR) the sample were rotated $180^{\circ}$ after each passing. No lubrication was used among the rolls. The surface of the rolls was cleaned in certain intervals. Distortion angle was measured by giving certain identifiable marks (vertical lines) in the sample before ASR and after the rolling was finished, the deviation angle of the marked lines from ordinate was measured. The expression relating the microscopic shear strain $(\gamma)$ and the distortion angle is simple and is the following one ${ }^{5}$

$\gamma=\tan \beta$

The rolled sheets were annealed at about $280^{\circ} \mathrm{C}$ for 1 hour and then allowed it to cool near the furnace edge in order to avoid thermal shock. This temperature and time for the annealing was chosen in accordance with previous studies ${ }^{5}$ where it was reported to be the most effective one. After heat treatment, the rolled sheets were cut according to the ASTM standard tensile specimen with $10 \mathrm{~mm}$ width. The test was manifested using a universal tensile machine (UTM) named Shimadzu Autograph at a strain rate of $10^{-3} \mathrm{~s}^{-1}$. A non-contact video extensometer MFA-25 was used in order to measure the changes in the length and width. The test was performed for three different set of sample cut in the $0^{\circ}, 45^{\circ}$ and $90^{\circ}$ from the rolled sheet and for each case eight different samples were investigated in order to validate the reproducibility of the results. The crystallographic orientation of the grains was characterized by measuring $\{200\}$ and $\{111\}$ X-ray pole figures using a Philips X'pert diffractometer equipped with texture goniometer. Samples were polished until the middle of the thickness and the $\mathrm{Cu}$ $\mathrm{K} \alpha$ radiation were used in these measurements. The acquired data was processed by dedicated software using the MTEX MATLAB tool box ${ }^{8}$. From the processed pole figures, a set of 1000 weighted orientations were extracted and used to compute the $<\mathrm{M}>$ value, using VPCS model code developed by Lebenshon and Tome ${ }^{9-11}$. The dislocation microstructure developed in the samples after deformation was characterized using a $300 \mathrm{kV}$ Hitachi H-9000 transmission electron microscope (TEM). TEM foils parallel to the sheet plane were taken at the sample's mid-thickness location. The samples were prepared by mechanical polishing of both sides and electro-polished using a double jet thinner with dilute solution of $\mathrm{HNO}_{3}$ and methanol under $10 \mathrm{~V}$, until perforation occurred.

\section{RESULTS AND DISCUSSIONS}

In this study, the aim was to investigate whether asymmetric rolling can be used to improve the formability of pure aluminium thick sheets. Some other previous studies suggested that asymmetric rolling can be a problem in the case of thick specimens ${ }^{12}$ where it was reported that the ideal shear texture was difficult to obtain by maintaining the rolling direction. The concern was on determining the roll power and also the shear strain generation along the entire length of the thick sheets. However, it was observed here that, the shear lines were imposed and were inclined linearly (Fig. 1). This does not necessarily suggest the dominance of crystallographic texture but clearly gives indication that shear strain was imposed during asymmetric rolling. The entire test was done after rolling and annealing. It was reported 
previously that, annealing at $280^{\circ} \mathrm{C}$ for $1 \mathrm{hr}$ is very useful to annihilate the dislocations without allowing the material to recrystallize . $^{5}$
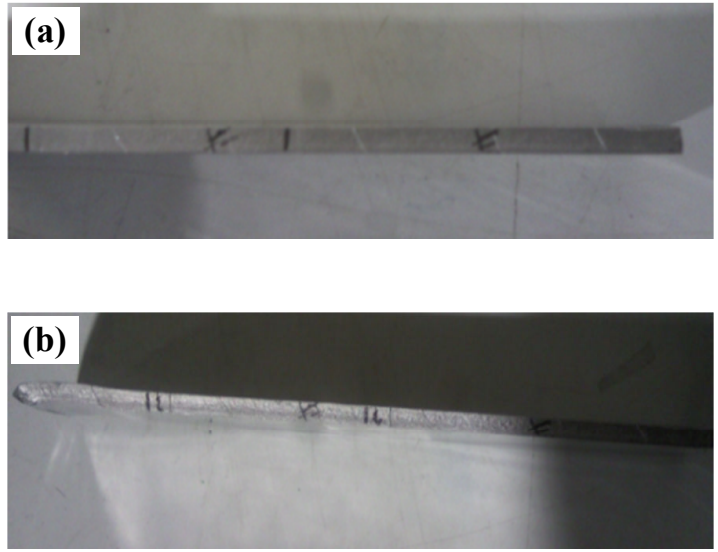

Figure 1. Macroscopic strain measurement; linear aligned marks resembling uniform strain distribution in the case of (a) ASRC after 6 pass,

(b) ASRR after 6 pass.

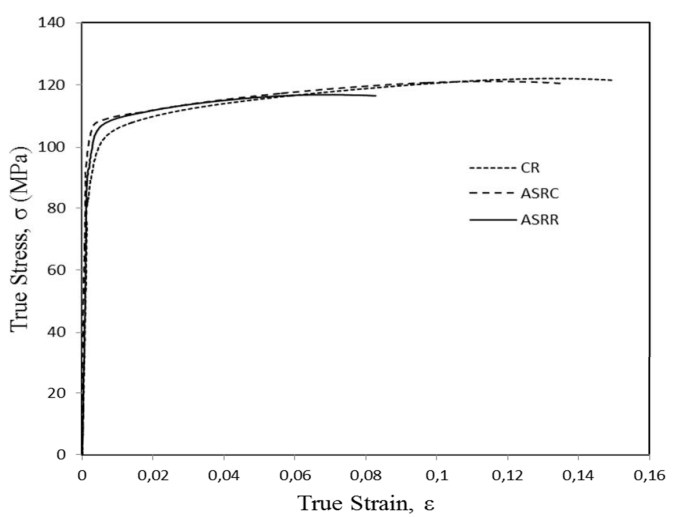

Figure 2. True stress and true strain curves for tensile specimens loaded at $0^{\circ}$.

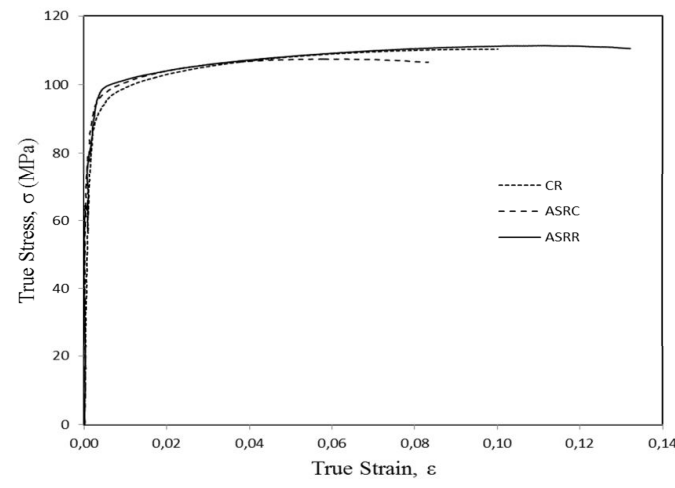

Figure 3. True stress and true strain curves for tensile specimens at $45^{\circ}$.

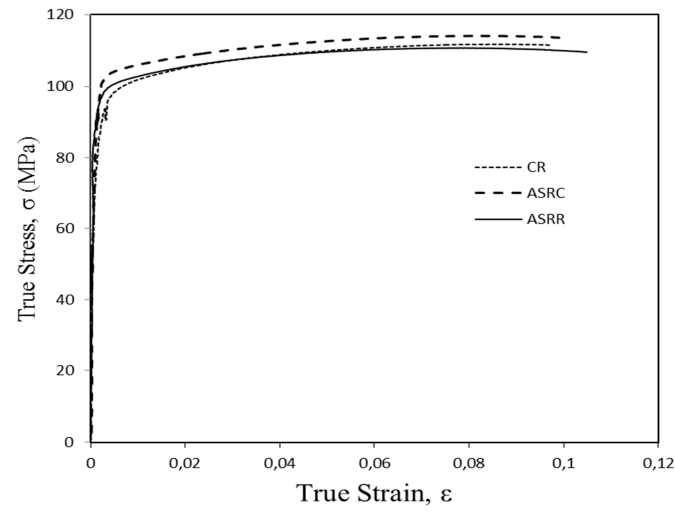

Figure 4. True stress and true strain curves for tensile specimens at $90^{\circ}$.

The mechanical behavior of the material is presented in Fig. 2-4. The important parameters are presented in Table 1-3. It was found that, the strain hardening behavior of CR, ASRC, ASRR are similar. At $0^{\circ}$, CR samples showed higher flow stress then ASRC and ASRR. At $45^{\circ}$ and $90^{\circ}$, ASRR and ASRC showed higher flow stress respectively. These differences in strain hardening behavior can have origin of the dislocation structure or/and crystallographic texture developed during rolling. ASRR samples showed higher strain value than ASRC in both $45^{\circ}$ and $90^{\circ}$ whereas for CR samples the value was in between.

Table 1. Yield stress $\left(\sigma_{\mathrm{o}}\right)$, Maximum stress $\left(\sigma_{\max }\right)$ and Uniform deformation $\left(\varepsilon_{\mathrm{u}}\right)$ loaded at $0^{\circ}$.

\begin{tabular}{|c|c|c|c|}
\hline Rolling & $\boldsymbol{\sigma}_{\mathbf{0}}(\mathbf{M P a})$ & $\boldsymbol{\sigma}_{\max }(\mathbf{M P a})$ & $\boldsymbol{\varepsilon}_{\mathbf{u}}$ \\
\hline $\mathbf{C R}$ & 106 & 122 & 0.147 \\
\hline ASRC & 108 & 121 & 0.133 \\
\hline ASRR & 102 & 117 & 0.081 \\
\hline
\end{tabular}

Table 2. Yield stress $\left(\sigma_{\mathrm{o}}\right)$, Maximum stress $\left(\sigma_{\max }\right)$ and Uniform deformation $\left(\varepsilon_{\mathrm{u}}\right)$ loaded at $45^{\circ}$.

\begin{tabular}{|c|c|c|c|}
\hline Rolling & $\boldsymbol{\sigma}_{\mathbf{0}}(\mathbf{M P a})$ & $\boldsymbol{\sigma}_{\max }(\mathbf{M P a})$ & $\boldsymbol{\varepsilon}_{\mathbf{u}}$ \\
\hline CR & 93 & 110 & 0.098 \\
\hline ASRC & 96 & 107 & 0.081 \\
\hline ASRR & 98 & 111 & 0.130 \\
\hline
\end{tabular}

Table 3. Yield stress $\left(\sigma_{\mathrm{o}}\right)$, Maximum stress $\left(\sigma_{\max }\right)$ and Uniform deformation $\left(\varepsilon_{\mathrm{u}}\right)$ loaded at $90^{\circ}$.

\begin{tabular}{|c|c|c|c|}
\hline Rolling & $\boldsymbol{\sigma}_{\mathbf{0}}(\mathbf{M P a})$ & $\boldsymbol{\sigma}_{\max }(\mathbf{M P a})$ & $\boldsymbol{\varepsilon}_{\mathbf{u}}$ \\
\hline CR & 98 & 112 & 0.095 \\
\hline ASRC & 104 & 114 & 0.098 \\
\hline ASRR & 101 & 110 & 0.103 \\
\hline
\end{tabular}

The influence of crystallographic texture for these strain hardening differences was investigated analyzing the $\sigma^{-\varepsilon}$ curves simulated and average Taylor factor $(<\mathrm{M}>$ values) extracted from pole figure (Fig. 5). The results from these calculations (Fig. 6) 
show that the differences in the initial crystal orientations
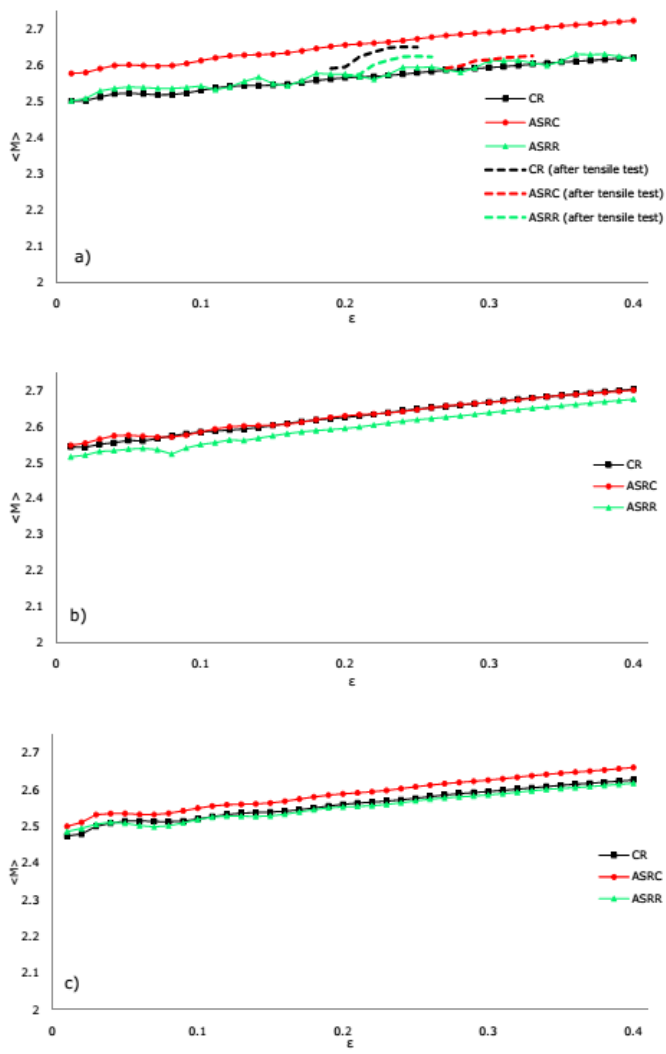

Figure 5. Evolution of Average Taylor Factor $(<\mathrm{M}>)$ during tensile test at (a) $0^{\circ}$, (b) $45^{\circ}$ and (c) $90^{\circ}$ of rolled and heat treated samples. Continuous lines - values calculated before tensile test; dashed lines - values calculated from pole figures after tensile test.

after CR, ASRC and ASRR have almost no influence on the flow stress and cannot explain the observed differences. The quality of the results computed with the VPSC model was evaluated for $\theta=0^{\circ}$ using two methods: comparing the average Taylor factor $(<\mathrm{M}>$ values) (Fig. 5.a) calculated from the experimental textures before (continues lines) and after (dashed lines) the tensile tests; and comparing the pole figures simulated with the model, and the texture measured after tensile test at $0^{\circ}$ (Fig. 7 to Fig. 9). For both methods a good agreement is observed, showing that VPSC model can be used to analyze the influence of crystallographic texture on $\sigma-\varepsilon$ curves.

The exclusion of the texture effect to explain the difference in the flow stress reveals that these differences should have microstructural origin. In Fig. 10 are presented the TEM observations for CR, ASRC and ASRR samples. The dislocation structure developed after heat treatment as well as after loading at $0^{\circ}$ in all samples is characterized by the presence of equiaxial dislocation cells (typical in rolled samples, due to activation of the large number of slip systems) with around 1-2 $\mu \mathrm{m}$ size, with many dislocations inside and relatively large crystallographic misorientations.
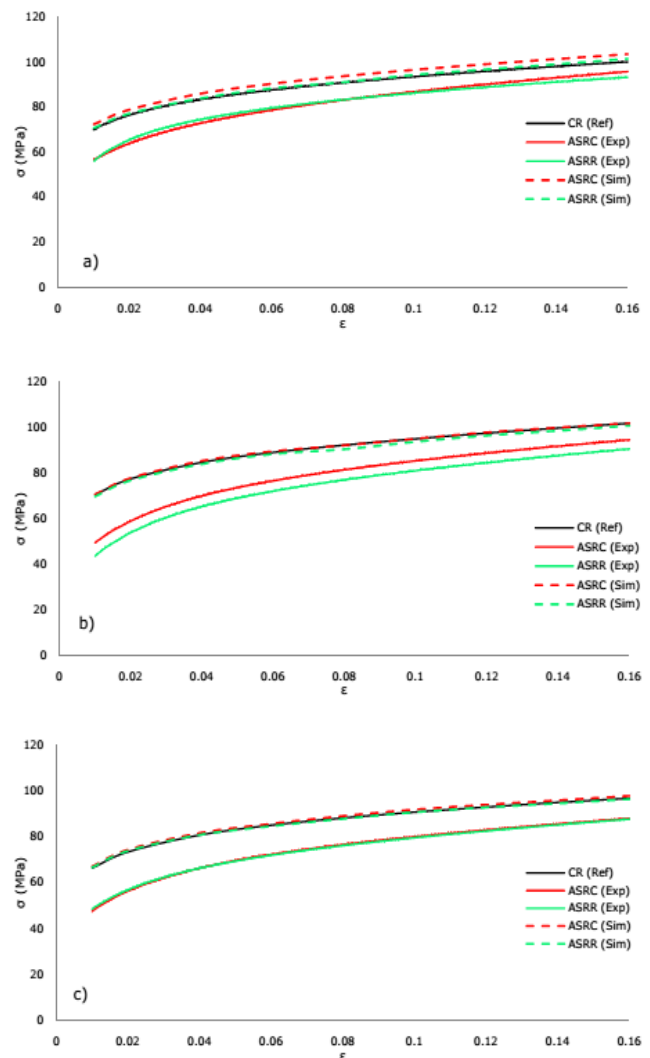

Figure 6. Experimental and simulated true stress-strain curves at (a) $0^{\circ}$, ( b) $45^{\circ}$ and (c) $90^{\circ}$. The CR curves were used as reference (Ref) in the simulations.

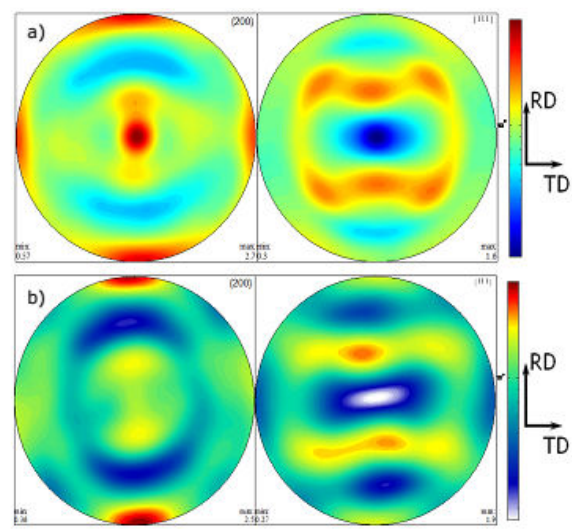

Figure 7. Experimental (a) and simulated (b) $\{200\}$ and $\{111\}$ pole figures for $\mathrm{CR}$ after tensile test at $0^{\circ}$.

From the comparison between these figures it is not possible to detect significant differences between dislocation microstructures that could explain the differences in $\sigma_{\max }$ presented by the samples after 
different rolling types. Therefore, other factors must be considered to explain these differences such as, changes in cell size and shape along the thickness and/or in crystallographic misorientations between neighbor cells ${ }^{12}$. Additional microstructural analysis which includes microstructural characterization in the thickness plane and measurements of sub grains misorientations should be performed to explain completely the strain hardening differences presented by CR, ASRC and ASRR samples. The sample preparation for the dislocation microstructure analysis in the perpendicular plane of the sheet is difficult due to the small thickness of the rolled samples (around $1 \mathrm{~mm}$ ) and the misorientation is complex to measure (requiring a point-to-point crystallographic orientation mapping) and compute its influence on the mechanical behavior of the sample.

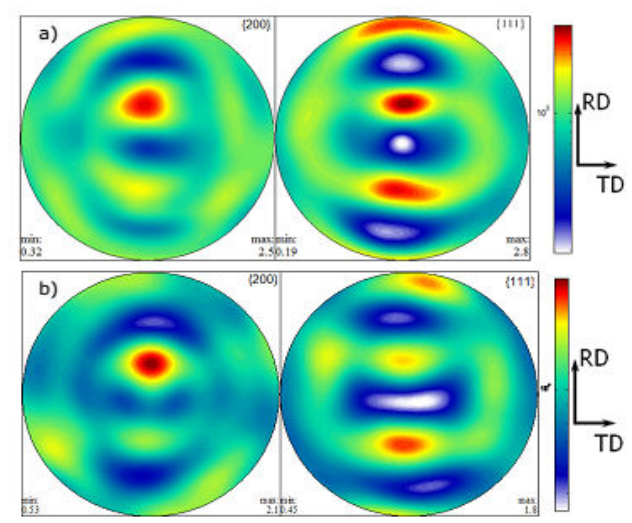

Figure 8. Experimental (a) and simulated (b) $\{200\}$ and $\{111\}$ pole figures for ASRC after tensile test at $0^{\circ}$.
The anisotropy coefficient of the CR, ASRC and ASRR samples are plotted in (Fig. 11) which takes into account the distribution of strain along the different dimensions of the specimen. The Normal and Planar coefficient of all the different rolling sequence is summarized in Table 4.

From these results it can be seen that ASRC and ASRR rolling gave a higher normal anisotropic coefficient when compared to $\mathrm{CR}$ samples. This is a clear indication of attainment of greater formability. It was also found that, ASRC samples showed higher value of normal anisotropy than ASRR. The highest normal anisotropy and the lowest planar anisotropy were observed by ASRC samples. So with this context in mind it can be said that ASRC can be done to improve the formability of pure aluminium sheets regardless the thickness.

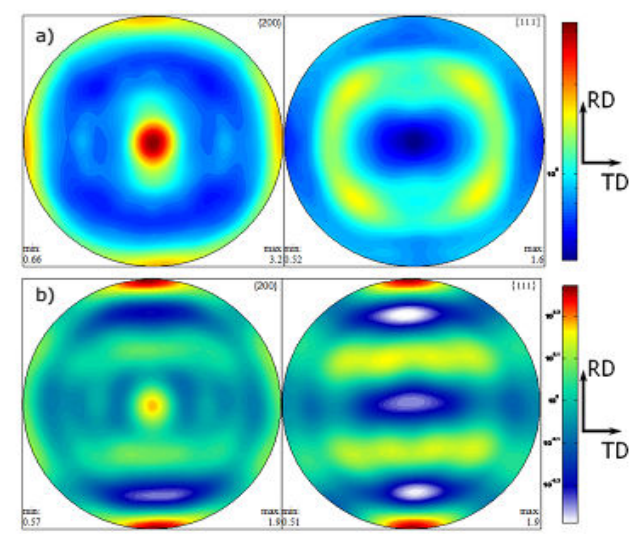

Figure 9. Experimental (a) and simulated (b) $\{200\}$ and $\{111\}$ pole figures for ASRR after tensile test at $0^{\circ}$.
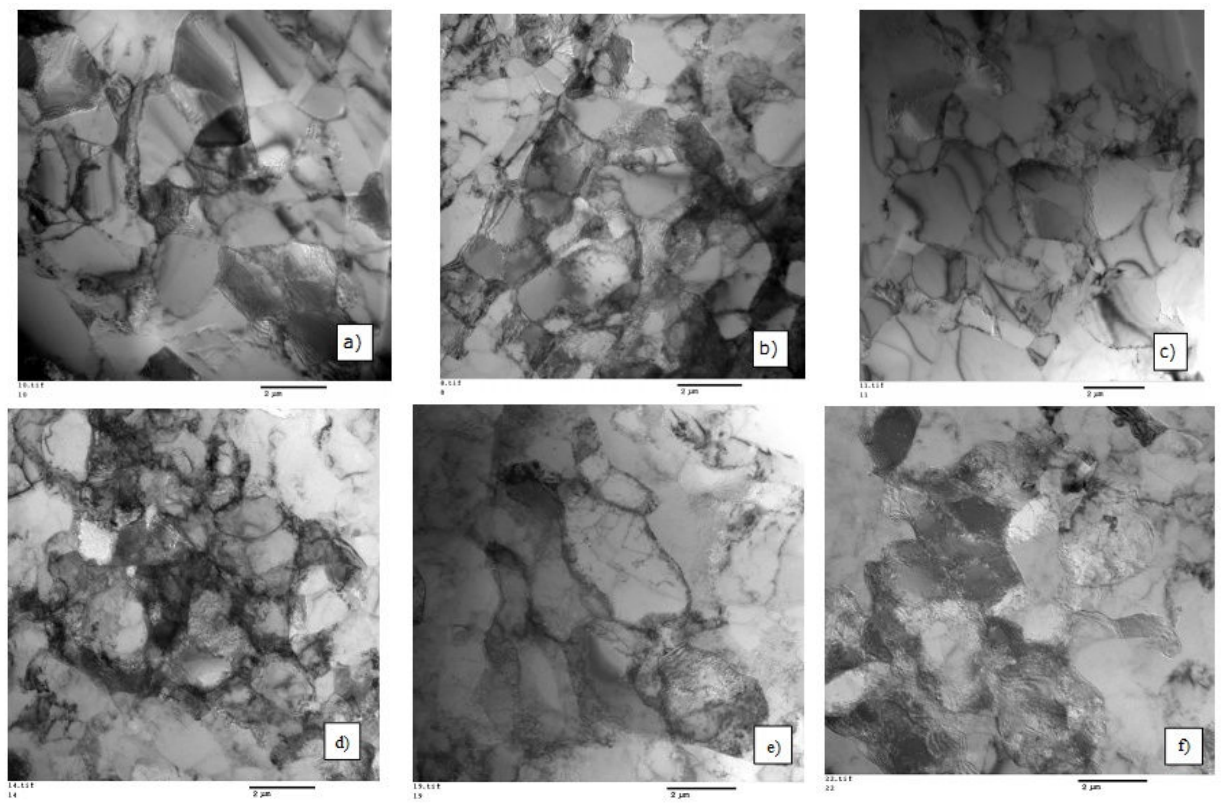

Figure 10. TEM image of the dislocation microstructure developed in (a) CR, (b) ASRC, (c) ASRR after heat treatment \& in (d) CR, (e) ASRC, (f) ASRR after loading at $0^{\circ}$.

Journal of Mechanical Engineering, Vol. ME 44, No. 2, December 2014

Transaction of the Mechanical Engineering Division, The Institution of Engineers, Bangladesh 


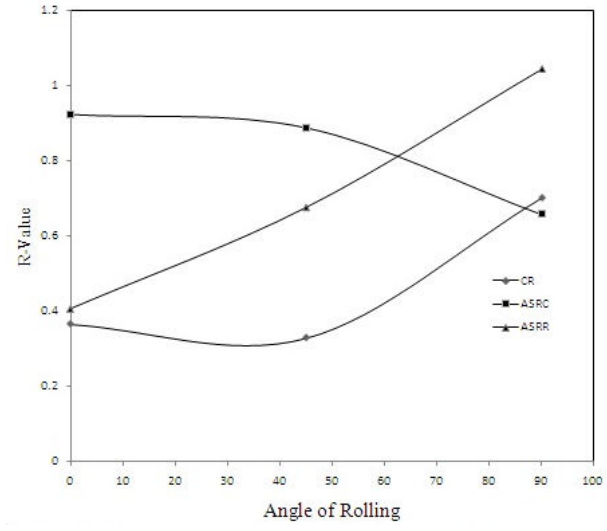

Figure 11. Anisotropic coefficient of CR, ASRC \& ASRR Samples.

Table 4. Anisotropic coefficients of CR, ASRC and ASRR samples.

\begin{tabular}{|c|c|c|c|c|}
\hline Specimen & $\boldsymbol{\theta}$ & $\mathbf{R}$ & $\begin{array}{c}\text { Normal } \\
/ \mathbf{R}\end{array}$ & $\begin{array}{c}\text { Planar } \\
\Delta \mathbf{R}\end{array}$ \\
\hline \multirow{3}{*}{ CR } & 0 & 0.3641 & \multirow{3}{*}{0.42965} & \multirow{3}{*}{0.2043} \\
\hline & 45 & 0.3275 & & \\
\hline & 90 & 0.6995 & & \\
\hline \multirow{3}{*}{ ASRC } & 0 & 0.9235 & \multirow{3}{*}{0.8387} & \multirow{3}{*}{-0.098} \\
\hline & 45 & 0.8877 & & \\
\hline & 90 & 0.6559 & & \\
\hline \multirow{3}{*}{ ASRR } & 0 & 0.405 & \multirow{3}{*}{0.6991} & \multirow{3}{*}{0.049} \\
\hline & 45 & 0.6736 & & \\
\hline & 90 & 1.0422 & & \\
\hline
\end{tabular}

\section{CONCLUSIONS}

The ASRC improved the normal anisotropy coefficient compared to CR specimens and also gave the lowest planar anisotropy coefficient. The shear strain that was imposed during the ASR was extensive and the shear marks was inclined more or less linearly without being zigzag or spiral lines. The highest normal anisotropy and the lowest planar anisotropy were observed by ASRC samples. From these results it can be said that asymmetric rolling especially ASRC can be used to improve the formability of the commercially pure aluminium irrespective of thickness. The texture analyses have shown, for asymmetrically rolled samples there is presence of crystals with orientation near to the ideal shear texture components. However, the intensities of these components were relatively low in both ASRC and ASRR samples.

\section{REFERENCES}

1. Dieter, G. E., 1988, "Mechanical Metallurgy", SI Metric Edition, McGraw-Hill Book Co.

2. Li D. and Ghosh K., 2004, "Biaxial warm forming behavior of aluminum sheet alloys" Materials Processing Technology Vol. 145(3), pp. 281-293.

3. Kocks U. F., Tome C. N. and. Wenk H. R,1998, "Texture and Anisotropy: Preferred Orientations in Polycrystals and their Effect on Materials Properties", Cambridge University Press.

4. Yu H., Tiue A. K., Lu C. Lie. X, Godbole A.and Kong C., 2013,"Mechanical properties of Al-Mg-Si alloy sheet $\mathrm{s}$ produced using asymmetric cryorolling and ageing treatment" Materials Science and Engineering, Vol. 568, pp. 212 - 218.

5. Lee J. K. and Lee D. N., 2008, “Texture control and grain refinement of AA1050 Al alloy sheets by asymmetric rolling" International Journal of Mechanical Sciences, Vol. 50(5), pp. $869-887$.

6. Karaman I., Sehitoglu H., Beaudoin A. J., Chumlyakov Y., Maier H. J. and Tome C. N., 2000, "Modeling the deformation behavior of Hadfield steel single and polycrystals due to twinning and slip" Acta Materialia, Vol. 48(9), pp. 2031-2047.

7. Austin, R. A. and. McDowell D. L., 2011, "A dislocation-based constitutive model for viscoplastic deformation of FCC metals at very high strain rates" International Journal of Plasticity, Vol. 27(1), pp.1-24. 8. Ding Y. and Jiang J., 2009, "Microstructures and mechanical properties of commercial purity iron processed by asymmetric rolling" Materials Science and Engineering: A Vol. 509(1-2), pp. 76-80.

9. Hielscher R. and Schaeben H., 2008, "A novel pole figure inversion method: specification of the MTEX algorithm" Journal of Applied Crystallography Vol. 41(6), pp. 1024-1037.

10. Lebensohn R. A. and Tomé C. N., 1993, "A self-consistent anisotropic approach for the simulation of plastic deformation and texture development of polycrystals: Application to zirconium alloys" Acta Metallurgica, Vol. 41(9), pp. 2611-2624.

11. Lebensohn R. A. and Tomé C. N., 1994, “A self-consistent viscoplastic model: prediction of rolling textures of anisotropic polycrystals" Materials Science and Engineering: A, Vol. 175(1-2), pp. 71-82.

12. Lee J. H. and Kim I., 2012, "Texture changes of asymmetrically rolled AA $1050 \mathrm{Al}$ alloy sheets" Materials Science, Vol. 702-703, pp. 332-335. 